\title{
Accuracy of Imaging in Preoperative Local Staging of Endometrial Cancer: Could Imaging Predict Low Risk Patients?
}

\author{
Maryam Rahmani $^{1 \times}$, Samira Heydari ${ }^{1}$, Azamsadat Mousavi ${ }^{2}$, Nasrin Ahmadinejad ${ }^{1}$, Shilan Azhdeh ${ }^{1}$, \\ Majid Shakiba ${ }^{1}$
}

\begin{abstract}
Objectives: In this study we aimed to evaluate diagnostic accuracy of magnetic resonance imaging (MRI) and transvaginal sonography (TVS) in identifying the depth of myometrial invasion and cervical involvement and also their relationship with the uterine arteries resistance index (RI) and pulsatility index (PI) in endometrial carcinoma.

Materials and Methods: We performed a prospective study on 45 women with histologically confirmed diagnosis of endometrial carcinoma. The study was performed from October 2009 to December 2012. All the patients were evaluated by 3T MRI and TVS and transvaginal color Doppler sonography of uterine arteries. All the patients underwent hysterectomy and the result of imaging and pathologic studies were compared.

Results: Mean age was $54.5 \pm 10.8$ years (33 to 77 years), mean gravid was $3.93 \pm 2.8$ and the mean parity was $3.9 \pm 2.5$. The pathology results indicated 29 patients (64.4\%) in stage IA and 16 patients (35.6\%) in stage IB. Mean endometrial thickness in stage IA patients was $18.4 \pm 14.4 \mathrm{~mm}$ and in stage IB patients was $38.5 \pm 11.5 \mathrm{~mm}$. TVS also showed positive predictive value (PPV) of $76.5 \%$, negative predictive value (NPV) of $88.9 \%$, sensitivity of $81.3 \%$, specificity of $85.7 \%$, and accuracy of $84.1 \%$ for assessment of the depth of myometrial invasion in endometrial carcinoma. We found PPV of $86.7 \%$, NPV of $92.3 \%$, sensitivity of $86.7 \%$, specificity of $92.3 \%$, and accuracy of $90.2 \%$ for MRI study.

Conclusions: TVS can evaluate the depth of myometrial invasion with an acceptable accuracy when MRI is not available or costeffective, or when MRI is contraindicated.

Both preoperative MRI and TVS can predict low risk patients (less than $50 \%$ of myometrial invasion) accurately; thereby avoiding lymphadenectomy in these patients.

Keywords: Endometrial cancer, Transvaginal sonography, Doppler ultrasonography, Magnetic resonance imaging, Myometrial invasion
\end{abstract}

\section{Introduction}

Endometrial cancer is the most common gynecological cancer in women and is considered the sixth most common malignancy in women $(1,2)$.

The incidence of endometrial carcinoma in Iran accounts for $17.6 \%$ of female genital tract carcinomas based on officially published female cancer statistics (3).

Surgical staging is the current way of endometrial cancer treatment; it includes total abdominal hysterectomy and bilateral salpingo-oophorectomy. Nowadays, there are controversies about routine lymphadenectomy.

Some studies suggest less extensive surgery for lowrisk patients, reserving para aortic lymphadenectomy for high-risk patients (4-6).

Several studies showed only $5 \%$ positive lymph node metastasis with superficial myometrial invasion (1,7-9).

Extra-uterine disease and lymph node metastasis cannot be evaluated via presurgical clinical examination; on the other hand, these are related to the depth of myometrial invasion, the most important and independent prognostic factor, cervical involvement and histopathological type and grade $(1,10,11)$.

Preoperative imaging assessment of myometrial invasion may help predict probable lymphadenopathies, especially in low grade tumors, and provide guidance for a better surgical approach in avoiding extensive surgery in low-risk patients with low-grade histopathology and myometrial invasion of less than $50 \%$.

Magnetic resonance imaging (MRI) and transvaginal sonography (TVS) are widely accepted as imaging choices in preoperative local staging of endometrial cancer (12) and accuracy of TVS in some recent studies was comparable with that of MRI, though there are still challenges $(8,13,14)$.

The use of Doppler sonography in the evaluation of endometrial cancer is a controversial issue (15).

In this prospective study, we evaluated the diagnostic accuracy of MRI, TVS and Doppler for detecting the 
depth of myometrial invasion and cervical involvement in endometrial carcinoma.

\section{Materials and Methods}

We performed a prospective study on 45 women with a confirmed diagnosis of endometrial carcinoma. The study was performed from October 2009 to December 2012 in a diagnostic and interventional radiology research center. Endometrial carcinoma was diagnosed via endometrial biopsy or curettage. All participants were evaluated with MRI, TVS, and Doppler. Inclusion criteria were diagnosis of endometrial carcinoma with endometrial curettage or biopsy. Patients who had never had sexual intercourse, patients who were not candidates for surgery due to advanced disease stage or poor clinical condition, and patients with contraindications for using MRI or using gadolinium during MRI were excluded. Patients underwent hysterectomy and bilateral salpingooophorectomy with lymph node dissection. The results of preoperative imaging and final histopathology of myometrial and cervical invasion were compared.

\section{Ultrasound}

A radiologist experienced in imaging women's reproductive systems performed sonography using a Medisone Accuvix V20 ultrasound unit by transvaginal transducer (ER4-9/10ED). All the patients underwent TVS with an empty bladder and uterus, viewed in the sagittal and coronal planes. The largest anteroposterior diameter of endometrial thickness in the mid sagittal plane was measured. The endometrial-myometrial junction (EMJ) was also examined and reported as being regular or irregular. The depth of myometrial invasion was evaluated and reported as less than $50 \%$ or more than $50 \%$ involvement. We divided the thinnest myometrial thickness in the involved area by normal appearing myometrium thickness to estimate the depth of myometrial invasion (Figure 1).

Color Doppler sonography of bilateral uterine arteries was performed at the level of the cervix, and the resistance index (RI) and pulsatility index (PI) of both sides were measured using the auto trace method.

\section{MRI Study}

The patients were asked to void one hour before the MRI to ensure a partially full bladder. All the patients fasted for at least 5-6 hours before the procedure, and a dose of $20 \mathrm{mg}$ hyoscine (IM) was administered to reduce artifacts caused by peristalsis (in the patients without contraindications). All imaging was performed in the supine position. The MRI studies were conducted with a 3-Tesla superconductive magnet (Siemens, MAGNETOM Trio, Germany) using a local pelvic phase arrayed coil. Turbo spin echo T2-weighted Images $(\mathrm{TR} / \mathrm{TE}=4000 / 101)$ with NSA $=2$, matrix size of $512 * 512, \mathrm{FOV}=250 \mathrm{~mm}$ and section thickness $=4 \mathrm{~mm}$ with intersection gap $=1 \mathrm{~mm}$ in

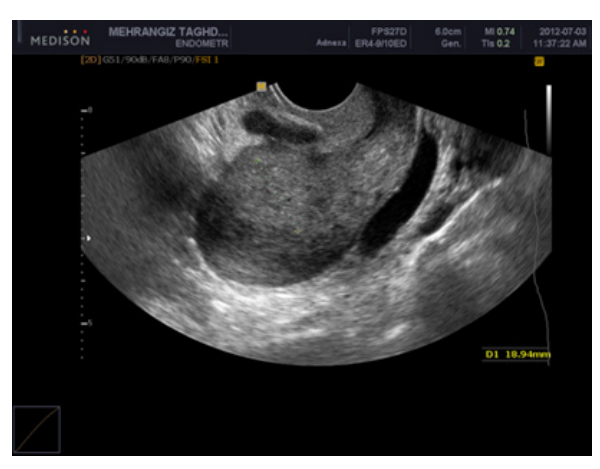

Figure 1. A 58-year-old Patient Presented With Postmenopausal Bleeding. Endometrial biopsy revealed endometrial cancer. Sagittal transvaginal sonography showed heterogeneous endometrial mass with AP diameter about $11 \mathrm{~mm}$, and anterior myometrial invasion of more than $50 \%$ was detected.

sagittal, coronal oblique and axial oblique (long axis and short axis of uterine cavity) planes were performed. Axial T1 images $(\mathrm{TR} / \mathrm{TE}=746 / 11)$ with 3-5 mm slice thickness and intersection gap $=1 \mathrm{~mm}$, matrix $256 \times 256$, and NSA = 2 were done. Contrast-enhanced MRI was performed after injection of $0.2 \mathrm{mmol} / \mathrm{kg}$ Gad-DTPA at a rate of $2 \mathrm{cc} / \mathrm{kg}$, with a scan delay of $2 \mathrm{~min} \pm 30 \mathrm{~s}$ (the time of maximal tumor-myometrial contrast) in sagittal and axial oblique sections by application of T1_VIBE_FS sequences $(\mathrm{TR} / \mathrm{TE}=4.74 / 2.18)$ with slice thickness of $3 \mathrm{~mm}$ and $\mathrm{FOV}=200 \mathrm{~mm}$.

MRI images were interpreted by a radiologist with expertise in pelvic MRI and blind to our findings and the final histopathologic grade of tumor (Figure 2).

All the patients underwent surgery and the depth of myometrial invasion and cervical involvement was determined through a pathologic study.

\section{Statistical Analysis}

The results of imaging were compared with the surgical pathologic results of myometrial invasion as the gold standard.

Statistical analysis was performed using SPSS software. Normally distributed continuous variables were compared between the patients with and without $50 \%$ of cancer invasion. A kappa test was used to study the association between the MRI and sonography. Positive predictive value (PPV) and negative predictive value (NPV), sensitivity, specificity, and accuracy were also used to describe the studied variables.

\section{Results}

Of 45 total patients, the mean age was $54.5 \pm 10.8$ years (33-77 years). The mean gravid and parity were $3.93 \pm 2.8$ [0-11] and 3.9 $\pm 2.5[0-9]$ respectively. No patient had a positive history of tamoxifen or HRT treatment. In all, $47.7 \%$ [21 of 44 ] had hypertension and $42.2 \%$ had type II diabetes [19 of 45]. Twenty-eight (62.2\%) patients 

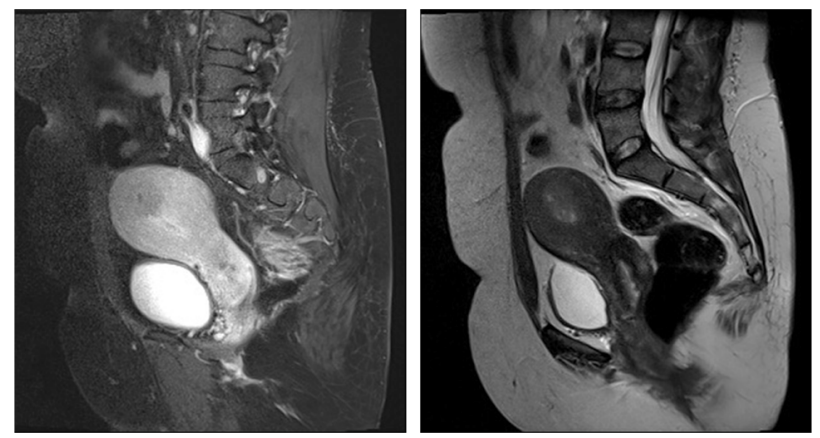

Figure 2. A 48-year-old Patient With AUB. D\&C revealed endometrial cancer. DCE pelvic MRI was performed, a: Fatsuppressed post-contrast sagittal T1 weighted image, and b: Sagittal T2 weighted image showing an endometrial mass associated with indistinct endometrial-myometrial junction (EMJ), suggestive for posterior myometrium invasion about $50 \%$. No serosal involvement was seen.

were postmenopausal. Forty-one $(93.2 \%, 41$ of 44$)$ patients had a positive history of vaginal bleeding. The most common histological grade was grade $1(57.8 \%$, 26 of 42). Histopathological evaluation showed that 29 (64.4\%) patients had less than 50\% myometrial invasion (superficial myometrial invasion, SMI) and $16(35.6 \%)$ had more than 50\% myometrial invasion (deep myometrial invasion, DMI). The most common histological type was endometroid (72.8\%, 32 of 44;). Table 1 displays the distribution of the different histological grades and types of tumors among the patients.

Mean endometrial thickness, measured by TVS, was $18.4 \pm 14.4 \mathrm{~mm}$ in the patients with SMI, determined in

Table 1. Histological Characteristics of Tumors

\begin{tabular}{llll}
\hline $\begin{array}{l}\text { Myometrial invasion } \\
\text { (in Histopathology) }\end{array}$ & $\begin{array}{l}\text { Superficial } \\
(<50 \%) \\
\text { Deep }(>50 \%)\end{array}$ & $\begin{array}{l}29(64.4 \%) \\
16(35.6 \%)\end{array}$ \\
\hline Histological & 1 & $26(61.9 \%)$ & \\
grade & 2 & $9(21.4 \%)$ & \\
& 3 & $7(16.7 \%)$ & \\
Histological & Serous & $2(4.5 \%)$ & \\
type & Adeno-squamous & $2(4.5 \%)$ \\
& Other & $8(18.2 \%)$ & \\
\hline
\end{tabular}

histopathology, and $38.5 \pm 11.5 \mathrm{~mm}$ in the patients with DMI $(P<0.0001)$. Determining the depth of myometrial invasion by TVS showed $84.1 \%$ of patients were correctly classified (37 of 44 patients). In fact, the sensitivity of sonography to correctly detect DMI was $81.3 \%$ and its specificity was $85.7 \%$. Other diagnostic indices of TVS in detecting the depth of myometrial invasion are shown in Table 2. Evaluation of the endometrial-myometrial interface in 5 patients was indeterminate. Among the patients with irregular endometrial-myometrial interface, $52.4 \%$ (11 of 21) showed DMI in histopathology; while in the patients with regular endometrial-myometrial interface, $15.8 \%$ (3 of 19) showed DMI in histopathology $(P$ value $=0.015$; odds ratio $[\mathrm{OR}]=5.9,95 \% \mathrm{CI}=1.3-26.3)$.

Comparison of Doppler indices in the patients with deep versus superficial myometrial invasion showed the means of all indices (including minimum RI, maximum RI, mean RI, RI difference, minimum PI, maximum PI, mean PI and PI difference) were statistically insignificant between deep and superficial tumors (all $P$ values greater than 0.05) (Table 3). All of the ROC curves for the differentiation of deep tumors based on Doppler indices were efficient.

MRI correctly determined the depth of myometrial invasion in $90.2 \%$ of the patients (37 of 41 ). Sensitivity of MRI in detection of deep invasion was $86.7 \%$ and its specificity was $92.3 \%$. Other MRI indices are shown in Table 2.

In comparing the diagnostic accuracy of MRI and TVS, there was no statistically significant difference $(P$ value $=0.29)$ (Figure 3 ).

Information of cervical invasion was only obtained for 30 patients in pathology. TVS correctly assessed cervical invasion in $28(93.3 \%)$ of these cases. Sensitivity and specificity of TVS in cervical invasion were $88.9 \%$ and $95.2 \%$, respectively. MRI correctly predicted cervical invasion in $26(96.3 \%)$ of the patients with $88.9 \%$ sensitivity and $100.0 \%$ specificity (Table 4). Comparing the diagnostic accuracy of MRI and TVS for cervical invasion showed no statistically significant difference $(P$ value $=0.75$ ) (Figure 4 ).

Comparing the mean of all Doppler indices between histological grades did not show any statistical difference.

Table 2. Diagnostic Indices of TVS, Doppler and MRI in Detection of DMI in Endometrial Carcinoma

\begin{tabular}{llcccccccc}
\hline & & Sen. (\%) & Spec. (\%) & PPV (\%) & NPV (\%) & PLR & NLR & Accur. (\%) & Kappa \\
\hline TVS & Estimation & 81.3 & 85.7 & 76.5 & 88.9 & 5.7 & 4.6 & 84.1 & 0.66 \\
TP=13, FN=3, TN=24, FP=4 & $95 \% \mathrm{Cl}$ & $54.4-96$ & $67.3-96$ & $50.1-93.2$ & $70.8-97.7$ & $2.2-14.5$ & $1.6-12.8$ & $69.9-93.4$ & $0.43-0.89$ \\
& Estimation & 93.8 & 48.2 & 51.7 & 92.9 & 1.8 & 7.72 & 65.1 & 0.36 \\
Doppler & $95 \% \mathrm{Cl}$ & $69.8-99.8$ & $28.7-68.1$ & $32.5-70.6$ & $66.1-99.8$ & $1.2-2.7$ & $1.1-53.5$ & $49.1-79$ & $0.14-0.58$ \\
$\mathrm{TP}=15, \mathrm{FN}=1, \mathrm{TN}=13, \mathrm{FP}=14$ & & 96.7 & 92.3 & 86.7 & 92.3 & 11.3 & 6.9 & 90.2 & 0.79 \\
$\mathrm{MRI}$ & Estimation & 95.9 & $74.9-99.1$ & $59.5-98.3$ & $74.9-99.1$ & $2.9-43.3$ & $1.9-25.3$ & $76.9-97.3$ & $0.59-0.99$ \\
\hline $\mathrm{TP}=13, \mathrm{FN}=2, \mathrm{TN}=24, \mathrm{FP}=2$ & $95 \% \mathrm{Cl}$ & $59.5-98.63$ & 74.9 &
\end{tabular}

Abbreviations: Sen., sensitivity; Spec, specificity; Accur, accuracy; TP, true positive; FN, false negative; TN, true negative; FP, false positive; PPV, Positive predictive value; NPV, negative predictive value; MRI, magnetic resonance imaging; TVS, transvaginal sonography; PLR, positive linear relationship; NLR, negative linear relationship. 
Table 3. Comparison of Doppler Indices Between Deep and Superficial Tumors

\begin{tabular}{llll}
\hline & & Mean \pm SD & P Value \\
\hline Minimum RI & Superficial & $0.73 \pm 0.12$ & \multirow{2}{*}{0.30} \\
& Deep & $0.69 \pm 0.10$ & \\
Maximum RI & Superficial & $0.77 \pm 0.11$ & \multirow{2}{*}{0.46} \\
& Deep & $0.75 \pm 0.10$ & \\
Mean RI & Superficial & $0.75 \pm 0.11$ & \multirow{2}{*}{0.37} \\
RI difference & Deep & $0.72 \pm 0.10$ & \\
& Superficial & $0.04 \pm 0.04$ & \multirow{2}{*}{0.36} \\
Minimum PI & Deep & $0.06 \pm 0.05$ & \\
& Superficial & $1.74 \pm 0.81$ & \multirow{2}{*}{0.16} \\
Maximum PI & Deep & $1.41 \pm 0.49$ & \\
& Superficial & $2.06 \pm 0.10$ & \multirow{2}{*}{0.14} \\
Mean PI & Deep & $1.73 \pm 0.46$ & \\
PI difference & Superficial & $1.90 \pm 0.89$ & \multirow{2}{*}{0.12} \\
& Deep & $1.57 \pm 0.45$ & \multirow{2}{*}{0.91} \\
& Superficial & $0.33 \pm 0.35$ & \\
& Deep & $0.32 \pm 0.29$ & \\
& & &
\end{tabular}

In addition, comparing the mean of these indices between endometroid tumors versus other types and also between the patients with and without cervical invasion did not show any statistical difference (all $P$ values greater than 0.05).

\section{Discussion}

Preoperative knowledge of the depth of myometrial invasion and cervical involvement can influence the recommended surgical approach. Studies showed less than $50 \%$ myometrial invasion is related with only $5 \%$ lymph node metastasis, whereas the patients with more than $50 \%$ myometrial invasion had a higher rate of lymph node metastasis ( $18 \%$ to $30 \%$ ). However, FIGO recommends lymphadenectomy for all patients; this could cause complications such as leg lymphedema and DVT, and may have no proven influence on survival rate in low risk patients (IA). Therefore, many studies suggest less extensive surgery in these patients and reserve para aortic lymphadenectomy for high-risk patients (IB, II) (4-6).

Preoperative imaging modalities such as MRI and TVS are the first-line choices; however, challenges remain on the optimal imaging technique for the assessment of myometrial invasion and cervical involvement (16).

In the present study, TVS and MRI had acceptable accuracy, sensitivity and specificity in detecting the depth

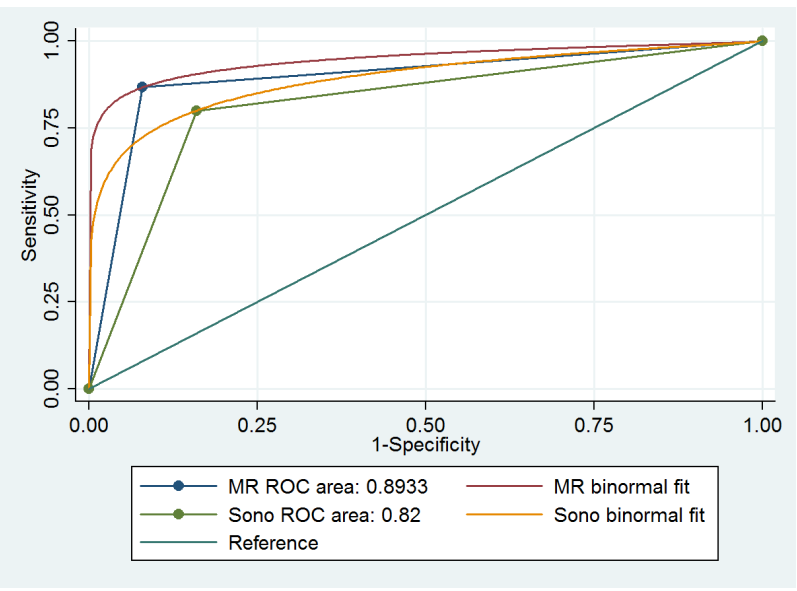

Figure 3. Comparing AUCs for ROC Curves of TVS Versus MRI in Detecting DMI.

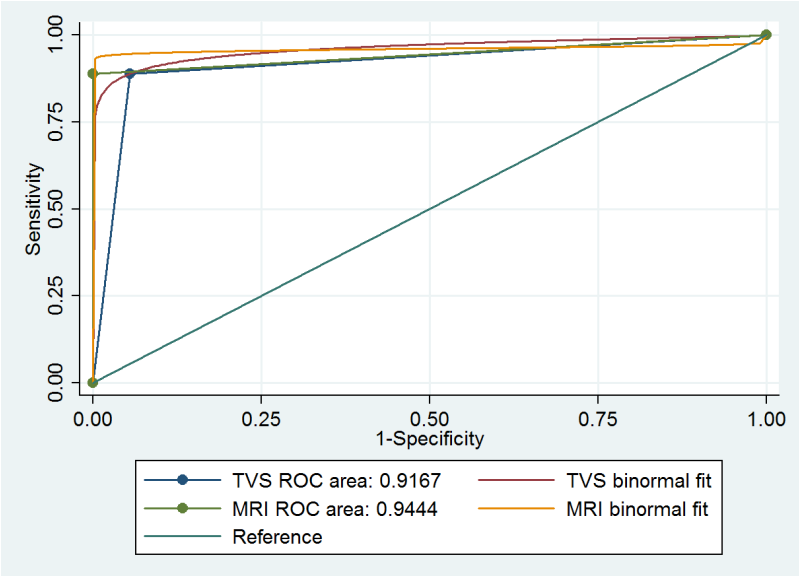

Figure 4. Comparing AUCs for ROC Curves of TVS Versus MRI in Detecting Cervical Invasion.

of myometrial invasion and cervical involvement, and TVS had comparable diagnostic values against MRI which was slightly more accurate.

In our study, endometrial thickness was a predictor for myometrial invasion; mean endometrial thickness in the patients with SMI was $18.4 \pm 14.4 \mathrm{~mm}$ and in the patients with DMI was $38.5 \pm 11.5 \mathrm{~mm}$ and this difference was significant at $P<0.0001$. In addition, regular EMJ was suggestive for no DMI $(P$ value $=0.015 ; \mathrm{OR}=5.9,95 \%$

Table 4. Diagnostic Indices of TVS and MRI in Detection of Cervical Invasion in Endometrial Carcinoma

\begin{tabular}{|c|c|c|c|c|c|c|c|c|c|}
\hline & & Sen. (\%) & Spec. (\%) & PPV (\%) & NPV (\%) & PLR & NLR & Accur. (\%) & Kappa \\
\hline TVS & Estimation & 88.9 & 95.2 & 88.9 & 95.2 & 18.7 & 8.6 & 93.3 & 0.84 \\
\hline$T P=8, F N=1, T N=20, F P=1$ & $95 \% \mathrm{Cl}$ & $51.8-99.7$ & $76.2-99.9$ & $51.8-99.7$ & $76.2-99.9$ & $2.7-128.2$ & $1.3-54.5$ & $77.9-99.2$ & $0.63-1$ \\
\hline MRI & Estimation & 88.9 & 100 & 100 & 94.7 & - & 9 & 96.3 & 0.91 \\
\hline $\mathrm{TP}=8, \mathrm{FN}=1, \mathrm{TN}=18, \mathrm{FP}=0$ & $95 \% \mathrm{Cl}$ & $51.8-99.7$ & $81.5-100$ & $63.1-100$ & $74-99.9$ & - & $1.4-57.1$ & 81-99.9 & $0.75-1$ \\
\hline
\end{tabular}

Abbreviations: Sen., sensitivity; Spec, specificity; Accur, accuracy; TP, true positive; FN, false negative; TN, true negative; FP, false positive; PPV, Positive predictive value; NPV, negative predictive value; MRI, magnetic resonance imaging; TVS, transvaginal sonography; PLR, positive linear relationship; NLR, negative linear relationship. 
$\mathrm{CI}=1.3-26.3$ ).

In predicting myometrial invasion, TVS had $81.3 \%$ sensitivity and $85.7 \%$ specificity.

The results of our study were equivalent to previous studies; MRI could identify the depth of myometrial invasion with $86.7 \%$ sensitivity and $92.3 \%$ specificity.

The agreement of TVS and MRI in detecting DMI was 0.74 ( $P$ value $\leq 0.001,95 \% \mathrm{CI}=0.52-0.95)$.

This study showed that RI and PI of uterine arteries were not significantly different between the patients with more and less than $50 \%$ of myometrial invasion (all $P$ values greater than 0.05 ; Table 3 ).

A systematic review showed a significant controversy in the sensitivity and specificity of TVS in previous studies (17). However, many studies showed similar performance for MRI and TVS (12).

A previous study showed DMI (low-risk and highrisk features of uterus) with accuracies of $78 \%-87 \%$ and sensitivity and specificity of $72 \%$ and $77 \%$, respectively (18).

Savelli et al demonstrated that when TVS was carried out by an expert radiologist, it showed acceptable accuracy in determining the depth of myometrial invasion and performed equally for MRI in preoperative staging (12).

We showed sensitivity and specificity of MRI in the prediction of myometrial invasion in premenopausal patients were in accordance with previous studies; however in postmenopausal women, sensitivity (100\%) was better than specificity (55.56\%), which could be due to extreme thinning of myometrium; though, PPV was significantly higher (10).

Studies showed that contrast-enhanced MRI had 90.9\% sensitivity, $91.8 \%$ specificity and $91.6 \%$ diagnostic accuracy for demonstrating DMI and the main causes of missed diagnosis were myomas, large tumor size, corneal lesion, adenomyosis, and non-endometroid tumor types (19).

Christensen et al showed that preoperative endometrial biopsy and MRI could spare 8 of 10 low-risk women from extended surgery and lymphadenectomy (18).

Another study also suggested that MRI could accurately identify low-risk patients and avoid unnecessary lymphadenectomy (20).

In our study, MRI and TVS had an accuracy of $93.3 \%$ and $96.3 \%$, respectively, in detecting cervical invasion. Sensitivity and specificity of TVS in cervical invasion were $88.9 \%$ and $95.2 \%$, respectively, and MRI had $88.9 \%$ sensitivity and $100 \%$ specificity (Table 4 ).

Comparing the diagnostic accuracy of MRI and TVS for cervical invasion showed no statistically significant difference $(P$ value $=0.75)$ (Figure 4$)$. Agreement of TVS and MRI in detecting cervical invasion was 0.73 ( $P$ value $\leq 0.001,95 \% \mathrm{CI}=0.49-0.98$ ).

The study of Lin et al showed T2-weighted MRI and DCE-MRI had accuracy, sensitivity and specificity of
$87 \%, 58 \%$, and $95 \%$, respectively, in detection of cervical invasion, which was compatible with previous studies; however, they showed that DWI could significantly improve diagnostic accuracy for identifying cervical stromal invasion (21).

One limitation in this study was that all ultrasound exams were performed by one expert radiologist, so interobserver and intra-observer errors were not evaluated. However, we believe that experience is quite important in accurate evaluation of these patients especially in ultrasound exams as well as MRI. Another limitation was related to the patients with preexisting benign findings such as large, multiple myomas, and adenomyotic changes. In these patients, imaging estimates of myometrial invasion could be inaccurate as provided imaging data about endometrial-myometrial junction and real myometrial thickness were suboptimal. A combination of ultrasound and DCE MRI will help to these patients with an accurate estimation. Some postmenopausal patients with diffuse myometrial thinning were also another challenge.

In conclusion, MRI and TVS had almost equal performance in many patients in detecting myometrial invasion and cervical involvement, although MRI was superior to TVS. However, MRI is expensive and time consuming whereas TVS is more available, cost-effective, and easily performed; and when performed by an expert, it may accurately evaluate the depth of myometrial invasion, especially in low-risk patients.

\section{Conflict of Interests}

Authors declare that they have no conflict of interests.

\section{Ethical Issues}

This study was approved by the Ethics Committee of Tehran University of Medical Sciences (Number 91-0298-18276-74499).

\section{Financial Support}

None.

\section{References}

1. Andreano A, Rechichi G, Rebora P, Sironi S, Valsecchi MG, Galimberti S. MR diffusion imaging for preoperative staging of myometrial invasion in patients with endometrial cancer: a systematic review and meta-analysis. Eur Radiol. 2014;24(6):1327-1338. doi:10.1007/s00330-014-3139-4

2. Epstein E, Blomqvist L. Imaging in endometrial cancer. Best Pract Res Clin Obstet Gynaecol. 2014;28(5):721-739. doi:10.1016/j.bpobgyn.2014.04.007

3. Momtahen S, Kadivar M, Kazzazi AS, Gholipour F. Assessment of gynecologic malignancies: a multicenter study in Tehran (1995-2005). Indian J Cancer. 2009;46(3):226-230. doi:10.4103/0019-509x.52957

4. Antonsen SL, Jensen LN, Loft A, et al. MRI, PET/CT and ultrasound in the preoperative staging of endometrial cancer - a multicenter prospective comparative study. Gynecol Oncol. 2013;128(2):300-308. doi:10.1016/j. 
ygyno.2012.11.025

5. Kokcu A, Kurtoglu E, Celik H, Kefeli M, Tosun M, Onal M. Is Surgical Staging Necessary for Patients with Low-risk Endometrial Cancer? A Retrospective Clinical Analysis. Asian Pac J Cancer Prev. 2015;16(13):5331-5335.

6. Imai $\mathrm{K}$, Kato $\mathrm{H}$, Katayama $\mathrm{K}$, et al. A preoperative risk-scoring system to predict lymph node metastasis in endometrial cancer and stratify patients for lymphadenectomy. Gynecol Oncol. 2016;142(2):273-277. doi:10.1016/j.ygyno.2016.06.004

7. Rodriguez-Trujillo A, Martinez-Serrano MJ, MartinezRoman S, et al. Preoperative Assessment of Myometrial Invasion in Endometrial Cancer by $3 \mathrm{D}$ Ultrasound and Diffusion-Weighted Magnetic Resonance Imaging: A Comparative Study. Int J Gynecol Cancer. 2016;26(6):11051110. doi:10.1097/igc.0000000000000724

8. Angioli R, Plotti F, Capriglione S, et al. Preoperative local staging of endometrial cancer: the challenge of imaging techniques and serum biomarkers. Arch Gynecol Obstet. 2016;294(6):1291-1298. doi:10.1007/s00404-016-4181-Z

9. Savelli L, Testa AC, Mabrouk M, et al. A prospective blinded comparison of the accuracy of transvaginal sonography and frozen section in the assessment of myometrial invasion in endometrial cancer. Gynecol Oncol. 2012;124(3):549-552. doi:10.1016/j.ygyno.2011.11.016

10. Wu LM, Xu JR, Gu HY, Hua J, Haacke EM, Hu J. Predictive value of T2-weighted imaging and contrast-enhanced MR imaging in assessing myometrial invasion in endometrial cancer: a pooled analysis of prospective studies. Eur Radiol. 2013;23(2):435-449. doi:10.1007/s00330-012-2609-9

11. Cetinkaya K, Atalay F, Bacinoglu A. Risk factors of lymph node metastases with endometrial carcinoma. Asian Pac J Cancer Prev. 2014;15(15):6353-6356.

12. Savelli L, Ceccarini M, Ludovisi M, et al. Preoperative local staging of endometrial cancer: transvaginal sonography vs. magnetic resonance imaging. Ultrasound Obstet Gynecol. 2008;31(5):560-566. doi:10.1002/uog.5295

13. Fischerova D, Fruhauf F, Zikan M, et al. Factors affecting sonographic preoperative local staging of endometrial cancer. Ultrasound Obstet Gynecol. 2014;43(5):575-585. doi:10.1002/uog.13248
14. Van Holsbeke C, Ameye L, Testa AC, et al. Development and external validation of new ultrasound-based mathematical models for preoperative prediction of high-risk endometrial cancer. Ultrasound Obstet Gynecol. 2014;43(5):586-595. doi:10.1002/uog.13216

15. Ozdemir S, Celik C, Emlik D, Kiresi D, Esen H. Assessment of myometrial invasion in endometrial cancer by transvaginal sonography, Doppler ultrasonography, magnetic resonance imaging and frozen section. Int J Gynecol Cancer. 2009;19(6):1085-1090. doi:10.1111/ IGC.0b013e3181ad3eb6

16. Christensen JW, Dueholm M, Hansen ES, Marinovskij E, Lundorf E, Ortoft G. Assessment of myometrial invasion in endometrial cancer using three-dimensional ultrasound and magnetic resonance imaging. Acta Obstet Gynecol Scand. 2016;95(1):55-64. doi:10.1111/aogs.12806

17. Alcazar JL, Orozco R, Martinez-Astorquiza Corral T, et al. Transvaginal ultrasound for preoperative assessment of myometrial invasion in patients with endometrial cancer: a systematic review and meta-analysis. Ultrasound Obstet Gynecol. 2015;46(4):405-413. doi:10.1002/uog.14905

18. Ortoft G, Dueholm M, Mathiesen O, et al. Preoperative staging of endometrial cancer using TVS, MRI, and hysteroscopy. Acta Obstet Gynecol Scand. 2013;92(5):536545. doi:10.1111/aogs.12103

19. Teng F, Zhang YF, Wang YM, et al. Contrast-enhanced MRI in preoperative assessment of myometrial and cervical invasion, and lymph node metastasis: diagnostic value and error analysis in endometrial carcinoma. Acta Obstet Gynecol Scand. 2015;94(3):266-273. doi:10.1111/ aogs. 12570

20. Hahn HS, Song HS, Lee IH, etal. Magnetic resonanceimaging and intraoperative frozen sectioning for the evaluation of risk factors associated with lymph node metastasis in endometrial cancer. Int J Gynecol Cancer. 2013;23(8):14111416. doi:10.1097/IGC.0b013e3182a580d3

21. Lin G, Huang YT, Chao A, et al. Endometrial cancer with cervical stromal invasion: diagnostic accuracy of diffusionweighted and dynamic contrast enhanced MR imaging at 3T. Eur Radiol. 2017;27(5):1867-1876. doi:10.1007/s00330016-4583-0

(C) 2018 The Author (s); This is an open-access article distributed under the terms of the Creative Commons Attribution License (http://creativecommons.org/licenses/by/4.0), which permits unrestricted use, distribution, and reproduction in any medium, provided the original work is properly cited. 